\title{
On Korn's constant for thin cylindrical domains
}

\author{
Roberto Paroni* and Giuseppe Tomassetti ${ }^{\dagger}$
}

\begin{abstract}
We consider an $\varepsilon$-parametrized collection of cylinders of cross section $\varepsilon \omega$, where $\omega \subset \mathbb{R}^{2}$, and of fixed length $\ell$. By Korn's inequality, there exists a positive constant $K^{\varepsilon}$ such that $\int_{\Omega^{\varepsilon}}|\operatorname{sym} \nabla \boldsymbol{u}|^{2} \mathrm{~d}^{3} \boldsymbol{x} \geq K^{\varepsilon} \int_{\Omega^{\varepsilon}}|\nabla \boldsymbol{u}|^{2} \mathrm{~d}^{3} \boldsymbol{x}$ provided that $\boldsymbol{u} \in H^{1}\left(\Omega ; \mathbb{R}^{3}\right)$ satisfies a condition that rules out infinitesimal rotations. We show that $K^{\varepsilon} / \varepsilon^{2}$ converges to a strictly positive limit, and we characterize this limit in terms of certain parameters that depend on the geometry of $\omega$ and on $\ell$.
\end{abstract}

Keywords: Korn's constant, Thin structures, Linear elasticity, $\Gamma$-convergence, Elastic stability.

AMS classification: 74K10, 74B05, 49R05.

\section{Introduction and main results}

Let $\omega \subset \mathbb{R}^{2}$ and $\ell>0$. For $\varepsilon>0$ a small parameter, let $\Omega^{\varepsilon}$ denote the cylinder whose typical point has the form $\boldsymbol{x}=\left(x, x_{3}\right)$ with $x=\left(x_{1}, x_{2}\right) \in \varepsilon \omega$ and $x_{3} \in(0, \ell)$, that is,

$$
\Omega^{\varepsilon}:=\varepsilon \omega \times(0, \ell) .
$$

Consider the collection of continuously-differentiable displacement fields $\boldsymbol{u}(\boldsymbol{x})=$ $\left(u_{1}(\boldsymbol{x}), u_{2}(\boldsymbol{x}), u_{3}(\boldsymbol{x})\right)$ satisfying one of the following conditions:

$$
\begin{aligned}
& \boldsymbol{u}(\boldsymbol{x})=0 \text { for } x_{3}=0 \text { and } x_{3}=\ell, \\
& \boldsymbol{u}(\boldsymbol{x})=0 \text { for } x_{3}=0, \\
& \int_{\Omega^{\varepsilon}} \boldsymbol{u} \mathrm{d}^{3} \boldsymbol{x}=0, \text { and } \int_{\Omega^{\varepsilon}} \boldsymbol{W}(\boldsymbol{u}) \mathrm{d}^{3} \boldsymbol{x}=0,
\end{aligned}
$$

where $\boldsymbol{W}(\boldsymbol{u}):=\frac{1}{2}\left(\nabla \boldsymbol{u}-\nabla \boldsymbol{u}^{T}\right)$. Let us set $\boldsymbol{E}(\boldsymbol{u}):=\frac{1}{2}\left(\nabla \boldsymbol{u}+\nabla \boldsymbol{u}^{T}\right)$. Korn's inequality asserts that there exists a constant $K^{\varepsilon}>0$, that does not depend on $\boldsymbol{u}$, such that

$$
K^{\varepsilon} \int_{\Omega^{\varepsilon}}|\nabla \boldsymbol{u}|^{2} \mathrm{~d}^{3} \boldsymbol{x} \leq \int_{\Omega^{\varepsilon}}|\boldsymbol{E}(\boldsymbol{u})|^{2} \mathrm{~d}^{3} \boldsymbol{x} .
$$

*Dipartimento di Architettura e Pianificazione, Università degli Studi di Sassari, 07041 Alghero, Italy. email:paroni@uniss.it

†Dipartimento di Ingegneria Civile, Università degli Studi di Roma "Tor Vergata", 00133 Roma, Italy. email:tomassetti@ing.uniroma2.it 
Hereafter, $K^{\varepsilon}$ shall denote the largest constant satisfying (2), that is,

$$
K^{\varepsilon}:=\inf \mathcal{R}^{\varepsilon}(\boldsymbol{u}),
$$

where

$$
\mathcal{R}^{\varepsilon}(\boldsymbol{u}):=\frac{\int_{\Omega^{\varepsilon}}|\boldsymbol{E}(\boldsymbol{u})|^{2} \mathrm{~d}^{3} \boldsymbol{x}}{\int_{\Omega^{\varepsilon}}|\nabla \boldsymbol{u}|^{2} \mathrm{~d}^{3} \boldsymbol{x}}
$$

is Rayleigh's quotient, and where the infimum in (3) is taken over the set of displacements fields $\boldsymbol{u}$ satisfying one of the conditions $(d d)-(n n)$. For convenience, we shall refer to $K^{\varepsilon}$ as Korn's constant - although most references use this name for $1 / K^{\varepsilon}$. In this paper we are concerned with the asymptotic behavior of the scaled Korn's constant:

$$
\kappa^{\varepsilon}:=\frac{K^{\varepsilon}}{\varepsilon^{2}} .
$$

We show that there exists $\kappa>0$ such that

$$
\lim _{\varepsilon \rightarrow 0} \kappa^{\varepsilon}=\kappa,
$$

and, more importantly, we characterize $\kappa$ in terms of $\omega$ and $\ell$ for each of the cases $(d d)-(n n)$.

The depedence of $\kappa$ on $\omega$ is expressed in terms of four parameters: the area $|\omega|$, the moments of inertia

$$
J_{1}:=\int_{\omega} x_{2}^{2} \mathrm{~d}^{2} x, \quad J_{2}:=\int_{\omega} x_{1}^{2} \mathrm{~d}^{2} x
$$

and the torsional moment of inertia:

$$
J_{t}:=\inf _{\psi \in C^{1}(\bar{\omega})} \int_{\omega}\left|\nabla \psi+x^{\perp}\right|^{2} \mathrm{~d}^{2} x, \quad \text { with } \quad x^{\perp}:=\left(-x_{2}, x_{1}\right) .
$$

We remark that

$$
J_{t}=\int_{\omega}\left|\nabla \psi_{\mathrm{m}}+x^{\perp}\right|^{2} \mathrm{~d}^{2} x=\int_{\omega} \nabla \psi_{\mathrm{m}} \cdot x^{\perp}+|x|^{2} \mathrm{~d}^{2} x,
$$

where $\psi_{\mathrm{m}}$ is the unique solution of the Neumann problem:

$$
\begin{cases}\Delta \psi_{\mathrm{m}}=0 & \text { in } \omega, \\ \left(\nabla \psi_{\mathrm{m}}+x^{\perp}\right) \cdot \boldsymbol{\nu}=0 & \text { on } \partial \omega, \\ \int_{\omega} \psi_{\mathrm{m}} \mathrm{d}^{2} x=0 . & \end{cases}
$$

The four parameters $|\omega|, J_{1}, J_{2}$, and $J_{t}$ determine a characteristic length $\ell_{\#}$ (the subscript \# stands for $d d, d n$, or $n n$ ) in a way that depends on the particular case considered, according to the following formulas:

$$
\ell_{d d}^{2}=\frac{\pi^{2}}{2|\omega|} \frac{J_{t}}{2}, \quad \ell_{d n}^{2}=\frac{\pi^{2}}{8|\omega|} \min \left\{\frac{J_{t}}{2}, J_{1}, J_{2}\right\}, \quad \ell_{n n}^{2}=\frac{\pi^{2}}{2|\omega|} \min \left\{\frac{J_{t}}{2}, J_{1}, J_{2}\right\} .
$$


We show that

$$
\kappa=\left(\frac{\ell_{\#}}{\ell}\right)^{2}, \quad \#=d d, d n, n n
$$

Our approach is based on the variational characterization of $K^{\varepsilon}$ and on standard results from the theory of $\Gamma$-convergence. We proceed as follows: in Section 2 we reformulate the problem of minimizing the Rayleigh quotient $\mathcal{R}^{\varepsilon}$ as a variational problem on a function space that does not depend on $\varepsilon$ (see $(21)$ ), by mapping $\Omega^{\varepsilon}$ into the cylinder

$$
\Omega=\omega \times(0, \ell)
$$

in the same section, we prove a compactness result (Proposition 1) for a sequence of displacements satisfying a certain renormalization condition (see (24)), and we characterize the limit of any such sequence (Proposition 2); we use this characterization in Section 3 to identify the $\Gamma$-limit (Propositions 3 and 4) and to prove (6) via Theorem 1; in Section 4 we provide a general formula (see $(60)$ ) for the minima of the $\Gamma$ - limit. It turns out that one can equivalently compute $\kappa$ by minimizing

$$
\mathcal{R}(\boldsymbol{w}, \theta):=\frac{\int_{0}^{\ell} J_{1}\left(w_{1}^{\prime \prime}\right)^{2}+J_{2}\left(w_{2}^{\prime \prime}\right)^{2}+|\omega|\left(w_{3}^{\prime}\right)^{2}+\frac{J_{t}}{2}\left(\theta^{\prime}\right)^{2} \mathrm{~d} x_{3}}{2|\omega| \int_{0}^{\ell}\left(w_{1}^{\prime}\right)^{2}+\left(w_{2}^{\prime}\right)^{2}+\theta^{2} \mathrm{~d} x_{3}},
$$

where $\boldsymbol{w}=\left(w_{1}\left(x_{3}\right), w_{2}\left(x_{3}\right), w_{3}\left(x_{3}\right)\right)$ and $\theta=\theta\left(x_{3}\right)$, and where each prime superscript denotes differentiation. Minimizing $\mathcal{R}$ is of course much easier than minimizing $\mathcal{R}^{\varepsilon}$. This allows us to determine the explicit formulas (11), a task we carry out in Section 4.

Looking at (13), the connection with rod theory becomes apparent: the numerator of the right-hand side of (13) is the elastic energy of a slender beam undergoing flexure and torsion, provided that the shear and the Young modulus are set equal to 1 and 2, respectively. The functions $\boldsymbol{w}$ and $\vartheta$ are to be understood as, respectively, the displacement of the axis of the cylinder, and the rotation of the cross section. Indeed, the approach we use in this paper is similar to that adopted to justify beam theories by $\Gamma$-convergence, see for instance $[1,50]$. As a matter of fact, the analysis carried out in this paper can be repeated for thin-walled beams $[14,15]$, and also for plate-like domains by using the same approach that is used to justify standard plate theories, see for instance $[7,42]$ in the setting of simple materials, and $[43,44,49,35,36]$ in the setting of non-simple materials.

The inequality (2) was first stated by Korn [27], who provided a proof in two cases: when the displacement vanishes on the entire boundary of the domain, and when the average rotation is zero. Both cases exclude infinitesimal rotations from the collection of admissible displacements. In the first case, the proof of Korn's inequality is elementary, being based on integration by parts. The second case is much more complicated. Friedrichs had to provide another proof in [16], pointing out in the same paper that he was not able to verify Korn's original proof. Alternative proofs have been given by several other workers 
$[48,12,39,25,7,54]$, with generalizations to exponents $1<p<+\infty$ [31], to non-constant coefficients in the form $\left|\operatorname{sym}\left(\nabla \boldsymbol{u} \boldsymbol{F}_{\mathrm{p}}\right)\right|^{2}$, where $\boldsymbol{F}_{\mathrm{p}}$ is the plastic strain [34], and to nongradient (incompatible) tensorial fields [37, 38].

As extensively reviewed by Horgan [22], Korn's inequality has many applications in continuum mechanics. It provides the key estimate needed to establish the solvability of the boundary value problem of linear elastostatics [40,7], and it can be used to establish several global and pointwise bounds on its solutions [55]. Furthermore, Korn's inequality enters in the estimate of the critical load in elastic stability theory [21,20] and it is involved in the estimates of the rate of convergence for finite elements approximations, see for instance [5]. Needless to say, the accuracy of the bounds obtained in all these applications is contingent on the precise estimate of Korn's constant. The first such estimate was given by Bernstein and Toupin [2] for spheres and disks. For these two types of domains, the exact value of Korn's constant was computed by Payne and Weinberger [48]. In the planar case, the result of Payne and Weinberger was extended by Dafermos to circular rings [9].

Although the exact value of Korn's constant is known only in a few cases, a large variety of results is available concerning its dependence on the geometric properties of the domain. An estimate for star-shaped domains was derived in [26]. Estimates for thin domains, such as rods and plates, can be found, for instance, in $[24,32,1,7]$. Other estimates have been derived for domains obtained by joining rods and plates $[6,8,33,18,3,4]$. These results are of extreme importance for the mathematical justification of models of common use in the engineering practice.

As a general rule, Korn's inequality degenerates as the thickness of the domain tends to zero (unless one imposes some additional restrictions on the displacement, see for instance [29]). For domains like the ones defined in (1), it is known that $K^{\varepsilon}$ scales as $\varepsilon^{2}$. Indeed, the bound

$$
K^{\varepsilon} \leq C_{1} \varepsilon^{2}
$$

is readily established by using the ansatz $\boldsymbol{u}(\boldsymbol{x})=\left(w\left(x_{3}\right), 0,-x_{1} w^{\prime}\left(x_{3}\right)\right)$ in (3). This ansatz yields $\int_{\Omega^{\varepsilon}}|\boldsymbol{W}(\boldsymbol{u})|^{2} \mathrm{~d} x^{3}=O\left(\varepsilon^{2}\right)$, and $\int_{\Omega^{\varepsilon}}|\boldsymbol{E}(\boldsymbol{u})|^{2} \mathrm{~d} x^{3}=O\left(\varepsilon^{4}\right)$. Thus, roughly speaking, thinner bodies can accommodate rotations that are much larger than the strain.

A more careful analysis, see $[24,1]$, shows that the bound (14) is asymptotically optimal, in the sense that there exists a constant $C_{2}>0$ such that

$$
C_{2} \varepsilon^{2} \leq K^{\varepsilon} .
$$

Remarkably, the asymptotic estimates (14)-(15) hold also for thin plates (for a simple derivation, see [41]).

Among the above cited papers, the closest in spirit to ours is [26], where Kondrat'ev and Oleinik prove that for a domain $\Omega^{\varepsilon}$ as defined in (1), with $\omega$ a disk of unit radius, Korn's constant for the $(d n)$ case satisfies the inequality

$$
\frac{C_{\mathrm{ko}}}{\ell^{2}+\varepsilon^{2}} \leq \frac{K^{\varepsilon}}{\varepsilon^{2}}=\kappa^{\varepsilon},
$$


where $C_{\text {ko }}$ does not depend on $\varepsilon$ and $\ell$. From (11a) and (11b) we find, for this particular geometry, that

$$
\kappa=\frac{\pi^{2}}{32 \ell^{2}},
$$

and hence from (6), we deduce that $C_{\mathrm{ko}} \leq \pi^{2} / 32$.

The results presented in this paper were announced in [45].

\section{Scaling and compactness}

Through this paper, we assume $\omega$ to be open, bounded, connected, simply connected, and with Lipschitz-continuous boundary. Such regularity guarantees that Korn's inequality holds true [40] (counterexamples may be found in [19, 56]). The requirement that $\omega$ be simply connected guarantees that a planar irrotational vector field has a potential, a fact we use in the proof of Proposition 2. Without loss of generality, we also assume

$$
\int_{\omega} x_{1} x_{2} \mathrm{~d}^{2} x=0 \quad \text { and } \quad \int_{\omega} x_{\alpha} \mathrm{d}^{2} x=0 \quad \alpha=1,2 .
$$

The appropriate function space for the minimization of Rayleigh's quotient is:

$$
H_{\#}^{1}\left(\Omega^{\varepsilon} ; \mathbb{R}^{3}\right):=\left\{\boldsymbol{u} \in H^{1}\left(\Omega^{\varepsilon} ; \mathbb{R}^{3}\right): \boldsymbol{u} \text { satisfies }(\#)\right\}, \quad \#=d d, d n, n n .
$$

With this definition, we have:

$$
K^{\varepsilon}=\inf _{\substack{\boldsymbol{u} \in H_{\#}^{1}\left(\Omega^{\varepsilon} ; \mathbb{R}^{3}\right) \\ \boldsymbol{u} \neq 0}} \mathcal{R}^{\varepsilon}(\boldsymbol{u})
$$

We find it convenient to reformulate equation (18) by means of functions defined on the domain $\Omega$ specified by (12), and to rescale the in-plane components of the displacement. Namely, to each displacement $\boldsymbol{u} \in H_{\#}^{1}\left(\Omega^{\varepsilon} ; \mathbb{R}^{3}\right)$ we associate the scaled displacement $\boldsymbol{v} \in$ $H_{\#}^{1}\left(\Omega ; \mathbb{R}^{3}\right)$ defined by:

$$
\boldsymbol{v}(\boldsymbol{x})=\left(\begin{array}{c}
\varepsilon u_{1}\left(\boldsymbol{x}^{\varepsilon}\right) \\
\varepsilon u_{2}\left(\boldsymbol{x}^{\varepsilon}\right) \\
u_{3}\left(\boldsymbol{x}^{\varepsilon}\right)
\end{array}\right) \quad \text { where } \quad \boldsymbol{x}^{\varepsilon}=\left(\begin{array}{c}
\varepsilon x_{1} \\
\varepsilon x_{2} \\
x_{3}
\end{array}\right)
$$

It then follows that

$$
\nabla \boldsymbol{u}\left(\boldsymbol{x}^{\varepsilon}\right)=\nabla^{\varepsilon} \boldsymbol{v}(\boldsymbol{x})
$$

where

$$
\nabla^{\varepsilon} \boldsymbol{v}:=\left(\begin{array}{ccc}
\frac{1}{\varepsilon^{2}} \frac{\partial v_{1}}{\partial x_{1}} & \frac{1}{\varepsilon^{2}} \frac{\partial v_{1}}{\partial x_{2}} & \frac{1}{\varepsilon} \frac{\partial v_{1}}{\partial x_{3}} \\
\frac{1}{\varepsilon^{2}} \frac{\partial v_{2}}{\partial x_{1}} & \frac{1}{\varepsilon^{2}} \frac{\partial v_{2}}{\partial x_{2}} & \frac{1}{\varepsilon} \frac{\partial v_{2}}{\partial x_{3}} \\
\frac{1}{\varepsilon} \frac{\partial v_{3}}{\partial x_{1}} & \frac{1}{\varepsilon} \frac{\partial v_{3}}{\partial x_{2}} & \frac{\partial v_{3}}{\partial x_{3}}
\end{array}\right)
$$


A simple computation based on (18), on (5), and on (20) leads to

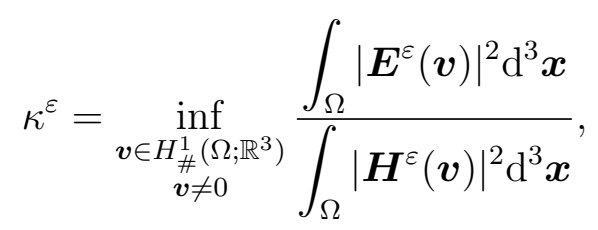

where

$$
\boldsymbol{E}^{\varepsilon}(\boldsymbol{v}):=\operatorname{sym} \nabla^{\varepsilon} \boldsymbol{v}, \quad \boldsymbol{H}^{\varepsilon}(\boldsymbol{v}):=\varepsilon \nabla^{\varepsilon} \boldsymbol{v} .
$$

We henceforth consider a sequence $\left\{\boldsymbol{v}^{\varepsilon}\right\} \subset H_{\#}^{1}\left(\Omega^{\varepsilon} ; \mathbb{R}^{3}\right)$ such that

$$
\frac{\int_{\Omega}\left|\boldsymbol{E}^{\varepsilon}\left(\boldsymbol{v}^{\varepsilon}\right)\right|^{2} \mathrm{~d}^{3} \boldsymbol{x}}{\int_{\Omega}\left|\boldsymbol{H}^{\varepsilon}\left(\boldsymbol{v}^{\varepsilon}\right)\right|^{2} \mathrm{~d}^{3} \boldsymbol{x}} \leq C .
$$

Note that, for whatever $\lambda^{\varepsilon} \neq 0,(23)$ is still satisfied if we replace $\boldsymbol{v}^{\varepsilon}$ with $\lambda^{\varepsilon} \boldsymbol{v}^{\varepsilon}$. Thus in order to have compactness for $\boldsymbol{v}^{\varepsilon}$ we need some renormalization condition. As renormalization condition we choose the following:

$$
\int_{\Omega}\left|\boldsymbol{H}^{\varepsilon}\left(\boldsymbol{v}^{\varepsilon}\right)\right|^{2} \mathrm{~d}^{3} \boldsymbol{x}=1 .
$$

The next proposition will be crucial in our analysis. The proof we provide draws upon [1] and $[17,13]$. In the statement below, we denote by $H_{\#}^{1}\left(\Omega ; \mathbb{R}_{\text {skw }}^{3 \times 3}\right)$ the Sobolev space of skew-matrix valued functions satisfying the same boundary conditions as $(d d)$ and $(d n)$ for the cases $\#=d d$ and $\#=d n$. For the remaining case we set

$$
H_{n n}^{1}\left(\Omega ; \mathbb{R}_{\mathrm{skw}}^{3 \times 3}\right)=\left\{\boldsymbol{W} \in H^{1}\left(\Omega ; \mathbb{R}_{\mathrm{skw}}^{3 \times 3}\right): \int_{\Omega} \boldsymbol{W} \mathrm{d}^{3} \boldsymbol{x}=0\right\} .
$$

Proposition 1 (Compactness). Let $\left\{\boldsymbol{v}^{\varepsilon}\right\}$ be a sequence satisfying the equi-boundedness condition (23) and the renormalization condition (24). Then there exist $\boldsymbol{v} \in H_{\#}^{1}\left(\Omega ; \mathbb{R}^{3}\right)$, $\boldsymbol{E} \in L^{2}\left(\Omega ; \mathbb{R}_{\mathrm{sym}}^{3 \times 3}\right)$, and $\boldsymbol{W} \in H_{\#}^{1}\left(\Omega ; \mathbb{R}_{\mathrm{skw}}^{3 \times 3}\right)$ such that

$$
\boldsymbol{v}^{\varepsilon} \stackrel{L^{2}}{\rightarrow} \boldsymbol{v}, \quad \boldsymbol{E}^{\varepsilon}\left(\boldsymbol{v}^{\varepsilon}\right) \stackrel{L^{2}}{\rightarrow} \boldsymbol{E}, \quad \boldsymbol{H}^{\varepsilon}\left(\boldsymbol{v}^{\varepsilon}\right) \stackrel{L^{2}}{\rightarrow} \boldsymbol{W}
$$

for some subsequence (not relabeled). Moreover,

$$
\frac{\partial \boldsymbol{W}}{\partial x_{\alpha}}=0
$$

Proof. We provide the proof for the case $\#=d d$, the other cases being similar. From (23)-(24) we have

$$
\int_{\Omega}\left|\boldsymbol{E}^{\varepsilon}\left(\boldsymbol{v}^{\varepsilon}\right)\right|^{2} \mathrm{~d}^{3} \boldsymbol{x} \leq C
$$


whence $(26)_{2}$. Moreover, for $\varepsilon$ sufficiently small, we have $\left\|\boldsymbol{E}\left(\boldsymbol{v}^{\varepsilon}\right)\right\|_{L^{2}(\Omega)} \leq\left\|\boldsymbol{E}^{\varepsilon}\left(\boldsymbol{v}^{\varepsilon}\right)\right\|_{L^{2}(\Omega)}$; thus, by (28) and by Korn's inequality (on the domain $\Omega$ ), $\sup _{\varepsilon}\left\|\boldsymbol{v}^{\varepsilon}\right\|_{H^{1}(\Omega)} \leq C$, and hence $(26)_{1}$ follows by weak compactness and by Rellich's theorem.

We next replace $\boldsymbol{v}^{\varepsilon}$ with its trivial extension to $\omega \times(-\infty,+\infty)$, and we introduce the shorthand notation:

$$
\boldsymbol{H}^{\varepsilon}(\boldsymbol{x}) \equiv\left(\boldsymbol{H}^{\varepsilon}\left(\boldsymbol{v}^{\varepsilon}\right)\right)(\boldsymbol{x})
$$

We begin by showing that for every $x_{3} \in(0, \ell)$ there exists a skew-symmetric matrix $\overline{\boldsymbol{W}}^{\varepsilon}\left(x_{3}\right)$ such that

$$
\int_{\omega} \int_{x_{3}-2 \varepsilon}^{x_{3}+2 \varepsilon}\left|\boldsymbol{H}^{\varepsilon}(\boldsymbol{y})-\overline{\boldsymbol{W}}^{\varepsilon}\left(x_{3}\right)\right|^{2} \mathrm{~d}^{3} \boldsymbol{y} \leq \varepsilon^{2} C \int_{\omega} \int_{x_{3}-2 \varepsilon}^{x_{3}+2 \varepsilon}\left|\boldsymbol{E}^{\varepsilon}\left(\boldsymbol{v}^{\varepsilon}\right)\right|^{2} \mathrm{~d}^{3} \boldsymbol{y}
$$

where $C$ is a constant independent on $\varepsilon$ and $x_{3}$. In order to establish (30), we consider the displacement field $\boldsymbol{u}^{\varepsilon} \in H^{1}\left(\varepsilon \omega \times(-\infty,+\infty) ; \mathbb{R}^{3}\right)$ defined by

$$
\boldsymbol{u}^{\varepsilon}\left(\boldsymbol{y}^{\varepsilon}\right)=\left(\begin{array}{c}
v_{1}^{\varepsilon}(\boldsymbol{y}) / \varepsilon \\
v_{2}^{\varepsilon}(\boldsymbol{y}) / \varepsilon \\
v_{3}^{\varepsilon}(\boldsymbol{y})
\end{array}\right) \quad \text { where } \quad \boldsymbol{y}^{\varepsilon}=\left(\begin{array}{c}
\varepsilon y_{1} \\
\varepsilon y_{2} \\
y_{3}
\end{array}\right)
$$

that is, by reversing the scaling (19). Since Korn's inequality is invariant under homothetic rescaling, there exists a constant $C$ independent on $\varepsilon$ such that

$$
\int_{\varepsilon \omega} \int_{x_{3}-2 \varepsilon}^{x_{3}+2 \varepsilon}\left|\nabla \boldsymbol{u}^{\varepsilon}\left(\boldsymbol{y}^{\varepsilon}\right)-\varepsilon^{-1} \overline{\boldsymbol{W}}^{\varepsilon}\left(x_{3}\right)\right|^{2} \mathrm{~d}^{3} \boldsymbol{y}^{\varepsilon} \leq C \int_{\varepsilon \omega} \int_{x_{3}-2 \varepsilon}^{x_{3}+2 \varepsilon}\left|\boldsymbol{E}\left(\boldsymbol{u}^{\varepsilon}\right)\right|^{2} \mathrm{~d}^{3} \boldsymbol{y}^{\varepsilon}
$$

where $\varepsilon^{-1} \overline{\boldsymbol{W}}^{\varepsilon}\left(x_{3}\right)=\frac{1}{4 \varepsilon^{3}|\omega|} \int_{\varepsilon \omega} \int_{x_{3}-2 \varepsilon}^{x_{3}+2 \varepsilon} \boldsymbol{W}\left(\boldsymbol{u}^{\varepsilon}\right) \mathrm{d}^{3} \boldsymbol{y}^{\varepsilon}$ is the average rotation in the slice $\varepsilon \omega \times$ $\left(x_{3}-2 \varepsilon, x_{3}+2 \varepsilon\right)$. By going back to the integration variable $\boldsymbol{y}$, and by recalling (20) and (22), we obtain

$$
\int_{\omega} \int_{x_{3}-2 \varepsilon}^{x_{3}+2 \varepsilon}\left|\varepsilon^{-1} \boldsymbol{H}^{\varepsilon}(\boldsymbol{y})-\varepsilon^{-1} \overline{\boldsymbol{W}^{\varepsilon}}\left(x_{3}\right)\right|^{2} \mathrm{~d}^{3} \boldsymbol{y} \leq C \int_{\omega} \int_{x_{3}-2 \varepsilon}^{x_{3}+2 \varepsilon}\left|\boldsymbol{E}^{\varepsilon}\left(\boldsymbol{v}^{\varepsilon}\right)\right|^{2} \mathrm{~d}^{3} \boldsymbol{y}
$$

whence (30).

We next define

$$
\overline{\boldsymbol{H}}^{\varepsilon}\left(x_{3}\right):=\frac{1}{|\omega|} \int_{\omega} \int_{-\infty}^{+\infty} \psi^{\varepsilon}\left(x_{3}-y_{3}\right) \boldsymbol{H}^{\varepsilon}(\boldsymbol{y}) \mathrm{d}^{3} \boldsymbol{y}
$$

where $\psi^{\varepsilon}\left(x_{3}\right)=\frac{1}{\varepsilon} \psi\left(\frac{x_{3}}{\varepsilon}\right)$, with $\psi$ a standard mollifier with support in $[-1,+1]$ and such that $\int_{-\infty}^{+\infty} \psi \mathrm{d} x_{3}=1$. On denoting by a prime superscript the derivative with respect to $x_{3}$, we have

$$
\left(\overline{\boldsymbol{H}}^{\varepsilon}\right)^{\prime}\left(x_{3}\right)=\frac{1}{|\omega|} \int_{\omega} \int_{-\infty}^{+\infty}\left(\psi^{\varepsilon}\right)^{\prime}\left(x_{3}-y_{3}\right) \boldsymbol{H}^{\varepsilon}(\boldsymbol{y}) \mathrm{d}^{3} \boldsymbol{y} .
$$


Since $\operatorname{supp}\left(\psi^{\varepsilon}\right) \subseteq[-\varepsilon,+\varepsilon]$ and $\int_{-\varepsilon}^{+\varepsilon}\left(\psi^{\varepsilon}\right)^{\prime} \mathrm{d} x_{3}=0$,

$$
\left(\overline{\boldsymbol{H}}^{\varepsilon}\right)^{\prime}\left(x_{3}\right)=\frac{1}{|\omega|} \int_{\omega} \int_{x_{3}-\varepsilon}^{x_{3}+\varepsilon}\left(\psi^{\varepsilon}\right)^{\prime}\left(x_{3}-y_{3}\right)\left(\boldsymbol{H}^{\varepsilon}(\boldsymbol{y})-\overline{\boldsymbol{W}}^{\varepsilon}\left(x_{3}\right)\right) \mathrm{d}^{3} \boldsymbol{y} .
$$

By using Hölder's inequality, noting that $\int_{-\varepsilon}^{+\varepsilon}\left|\left(\psi^{\varepsilon}\right)^{\prime}\right|^{2} \mathrm{~d} x_{3}=C \varepsilon^{-3}$, and recalling (30), we arrive at

$$
\left|\left(\overline{\boldsymbol{H}}^{\varepsilon}\right)^{\prime}\left(x_{3}\right)\right|^{2} \leq \frac{C}{\varepsilon^{3}} \int_{\omega} \int_{x_{3}-\varepsilon}^{x_{3}+\varepsilon}\left|\boldsymbol{H}^{\varepsilon}(\boldsymbol{y})-\overline{\boldsymbol{W}}^{\varepsilon}\left(x_{3}\right)\right|^{2} \mathrm{~d}^{3} \boldsymbol{y} \leq \frac{C}{\varepsilon} \int_{\omega} \int_{x_{3}-2 \varepsilon}^{x_{3}+2 \varepsilon}\left|\boldsymbol{E}^{\varepsilon}\left(\boldsymbol{u}^{\varepsilon}\right)\right|^{2} \mathrm{~d}^{3} \boldsymbol{y}
$$

From (32) we obtain:

$$
\int_{-\infty}^{+\infty}\left|\left(\overline{\boldsymbol{H}}^{\varepsilon}\right)^{\prime}\right|^{2} \mathrm{~d} x_{3} \leq C \int_{\Omega}\left|\boldsymbol{E}^{\varepsilon}\left(\boldsymbol{u}^{\varepsilon}\right)\right|^{2} \mathrm{~d}^{3} \boldsymbol{x}
$$

Since $\operatorname{supp}\left(\overline{\boldsymbol{H}}^{\varepsilon}\right) \subseteq(-\varepsilon, \ell+\varepsilon)$, it follows from (28) and (33) that $\overline{\boldsymbol{H}}^{\varepsilon}$ is bounded in $H^{1}\left(\mathbb{R} ; \mathbb{R}^{3 \times 3}\right)$, and that there exists a subsequence (not relabeled) such that

$$
\overline{\boldsymbol{H}}^{\varepsilon} \stackrel{L^{2}}{\rightarrow} \overline{\boldsymbol{W}}
$$

with $\overline{\boldsymbol{W}} \in H^{1}\left(\mathbb{R} ; \mathbb{R}^{3 \times 3}\right)$ satisfying

$$
\overline{\boldsymbol{W}}(0)=\overline{\boldsymbol{W}}(\ell)=0 .
$$

We next estimate:

$$
\begin{aligned}
\int_{\omega} \int_{x_{3}-\varepsilon}^{x_{3}+\varepsilon} & \left|\overline{\boldsymbol{H}}^{\varepsilon}\left(z_{3}\right)-\boldsymbol{H}^{\varepsilon}(\boldsymbol{z})\right|^{2} \mathrm{~d}^{3} \boldsymbol{z} \\
& \leq 2 \int_{\omega} \int_{x_{3}-\varepsilon}^{x_{3}+\varepsilon}\left|\overline{\boldsymbol{H}}^{\varepsilon}\left(z_{3}\right)-\overline{\boldsymbol{W}}^{\varepsilon}\left(x_{3}\right)\right|^{2} \mathrm{~d}^{3} \boldsymbol{z}+2 \int_{\omega} \int_{x_{3}-\varepsilon}^{x_{3}+\varepsilon}\left|\boldsymbol{H}^{\varepsilon}\left(z_{3}\right)-\overline{\boldsymbol{W}}^{\varepsilon}\left(x_{3}\right)\right|^{2} \mathrm{~d}^{3} \boldsymbol{z} .
\end{aligned}
$$

The second term on the right-hand side of (35) is immediately estimated using (30). We therefore turn our attention to the first term. Since $\int_{-\varepsilon}^{+\varepsilon} \psi^{\varepsilon} \mathrm{d} x_{3}=1$, we have, by (31),

$$
\overline{\boldsymbol{H}}^{\varepsilon}\left(z_{3}\right)-\overline{\boldsymbol{W}}^{\varepsilon}\left(x_{3}\right)=\frac{1}{|\omega|} \int_{\omega} \int_{z_{3}-\varepsilon}^{z_{3}+\varepsilon} \psi^{\varepsilon}\left(z_{3}-y_{3}\right)\left(\boldsymbol{H}^{\varepsilon}(\boldsymbol{y})-\overline{\boldsymbol{W}}^{\varepsilon}\left(x_{3}\right)\right) \mathrm{d}^{3} \boldsymbol{y} .
$$

On using Jensen's and Hölder's inequalities, and the fact that $\int_{-\varepsilon}^{+\varepsilon}\left|\psi^{\varepsilon}\left(x_{3}\right)\right|^{2} \mathrm{~d} x_{3}=C / \varepsilon$, we deduce that

$$
\left|\overline{\boldsymbol{H}}^{\varepsilon}\left(z_{3}\right)-\overline{\boldsymbol{W}}^{\varepsilon}\left(x_{3}\right)\right|^{2} \leq \frac{C}{\varepsilon} \int_{\omega} \int_{x_{3}-2 \varepsilon}^{x_{3}+2 \varepsilon}\left|\boldsymbol{H}^{\varepsilon}(\boldsymbol{y})-\overline{\boldsymbol{W}}^{\varepsilon}\left(x_{3}\right)\right|^{2} \mathrm{~d}^{3} \boldsymbol{y} \text { if } z_{3} \in\left(x_{3}-\varepsilon, x_{3}+\varepsilon\right),
$$

and hence,

$$
\int_{\omega} \int_{x_{3}-\varepsilon}^{x_{3}+\varepsilon}\left|\overline{\boldsymbol{H}}^{\varepsilon}\left(z_{3}\right)-\overline{\boldsymbol{W}}^{\varepsilon}\left(x_{3}\right)\right|^{2} \mathrm{~d}^{3} \boldsymbol{z} \leq C \int_{\omega} \int_{x_{3}-2 \varepsilon}^{x_{3}+2 \varepsilon}\left|\boldsymbol{H}^{\varepsilon}(\boldsymbol{z})-\overline{\boldsymbol{W}}^{\varepsilon}\left(x_{3}\right)\right|^{2} \mathrm{~d}^{3} \boldsymbol{z} .
$$


By (35), (37), and (30), we have

$$
\int_{\omega} \int_{x_{3}-\varepsilon}^{x_{3}+\varepsilon}\left|\overline{\boldsymbol{H}}^{\varepsilon}\left(z_{3}\right)-\boldsymbol{H}^{\varepsilon}(\boldsymbol{z})\right|^{2} \mathrm{~d}^{3} \boldsymbol{z} \leq C \varepsilon^{2} \int_{\omega} \int_{x_{3}-2 \varepsilon}^{x_{3}+2 \varepsilon}\left|\boldsymbol{E}^{\varepsilon}\left(\boldsymbol{u}^{\varepsilon}\right)\right|^{2} \mathrm{~d}^{3} \boldsymbol{z} .
$$

On integrating (38) with respect to $x_{3}$, recalling (28), and extending $\overline{\boldsymbol{H}}^{\varepsilon}$ in the following manner

$$
\overline{\boldsymbol{H}}^{\varepsilon}(\boldsymbol{x}):=\overline{\boldsymbol{H}}^{\varepsilon}\left(x_{3}\right) \text { for all } \boldsymbol{x} \in \Omega,
$$

we arrive at

$$
\left\|\overline{\boldsymbol{H}}^{\varepsilon}-\boldsymbol{H}^{\varepsilon}\right\|_{L^{2}(\Omega)} \rightarrow 0
$$

Thus, on letting

$$
\boldsymbol{W}(\boldsymbol{x}):=\overline{\boldsymbol{W}}\left(x_{3}\right) \text { for all } \boldsymbol{x} \in \Omega,
$$

the claimed strong convergence $(26)_{3}$ follows from (39) and (34).

Finally, since sym $\boldsymbol{H}^{\varepsilon}=\varepsilon \boldsymbol{E}^{\varepsilon}\left(\boldsymbol{v}^{\varepsilon}\right)$ converges strongly to zero in $L^{2}$, we have that sym $\boldsymbol{W}=$ 0 , and hence $\boldsymbol{W}$ is a function taking values in the space of skew-symmetric matrices.

We now characterize some of the components of the tensor fields $\boldsymbol{E}$ and $\boldsymbol{W}$ defined in Proposition 1.

Proposition 2 (Identification of the limit). Under the assumptions of Proposition 1, the limits $\boldsymbol{v}, \boldsymbol{E}$, and $\boldsymbol{W}$ in (26) satisfy:

$$
E_{\alpha i}(\boldsymbol{v})=0, \quad E_{33}(\boldsymbol{v})=E_{33}, \quad W_{\alpha 3}(\boldsymbol{v})=W_{\alpha 3},
$$

for $\alpha=1,2$ and $i=1,2,3$. Furthermore, there exists $\varphi \in L^{2}\left(0, \ell ; H^{1}(\omega)\right)$ such that

$$
\left(\begin{array}{l}
E_{13} \\
E_{23}
\end{array}\right)=\frac{1}{2}\left(x^{\perp} \frac{\partial W_{21}}{\partial x_{3}}+\nabla_{x} \varphi\right)
$$

where $\nabla_{x} \varphi=\left(\frac{\partial \varphi}{\partial x_{1}}, \frac{\partial \varphi}{\partial x_{2}}\right)$.

Proof. For $\varepsilon$ sufficiently small, we have $\left|E_{\alpha i}(\boldsymbol{v})\right| \leq \varepsilon\left|E_{\alpha i}^{\varepsilon}(\boldsymbol{v})\right|$, therefore, by $(26)_{2}$ and by lower semicontinuity,

$$
\left\|E_{\alpha i}(\boldsymbol{v})\right\|_{L^{2}(\Omega)} \leq \liminf _{\varepsilon \rightarrow 0}\left\|E_{\alpha i}\left(\boldsymbol{v}^{\varepsilon}\right)\right\|_{L^{2}(\Omega)} \leq \liminf _{\varepsilon \rightarrow 0} \varepsilon\left\|E_{\alpha i}^{\varepsilon}\left(\boldsymbol{v}^{\varepsilon}\right)\right\|_{L^{2}(\Omega)}=0,
$$

whence $(40)_{1}$. Next, $(40)_{2,3}$ follow by passing to the limit in the identities $E_{33}\left(\boldsymbol{v}^{\varepsilon}\right)=E_{33}^{\varepsilon}\left(\boldsymbol{v}^{\varepsilon}\right)$ and $\left(\operatorname{skw} \boldsymbol{H}^{\varepsilon}\left(\boldsymbol{v}^{\varepsilon}\right)\right)_{\alpha 3}=W_{\alpha 3}\left(\boldsymbol{v}^{\varepsilon}\right)$. Moreover, for $\boldsymbol{W}^{\varepsilon}\left(\boldsymbol{v}^{\varepsilon}\right):=\operatorname{skw} \boldsymbol{H}^{\varepsilon}\left(\boldsymbol{v}^{\varepsilon}\right)$, we have

$$
\frac{\partial W_{21}^{\varepsilon}\left(\boldsymbol{v}^{\varepsilon}\right)}{\partial x_{3}}=\frac{\partial E_{23}^{\varepsilon}\left(\boldsymbol{v}^{\varepsilon}\right)}{\partial x_{1}}-\frac{\partial E_{13}^{\varepsilon}\left(\boldsymbol{v}^{\varepsilon}\right)}{\partial x_{2}} \text { in } \mathcal{D}^{\prime}(\Omega),
$$

whence, by a passage to the limit,

$$
\frac{\partial W_{21}}{\partial x_{3}}=\frac{\partial E_{23}}{\partial x_{1}}-\frac{\partial E_{13}}{\partial x_{2}} \text { in } \mathcal{D}^{\prime}(\Omega) .
$$


Now, let $\gamma \in L^{2}\left(\Omega ; \mathbb{R}^{2}\right)$ be defined by

$$
\left(\begin{array}{l}
\gamma_{1} \\
\gamma_{2}
\end{array}\right)=2\left(\begin{array}{c}
E_{13} \\
E_{23}
\end{array}\right)-\left(\begin{array}{c}
-x_{2} \\
x_{1}
\end{array}\right) \frac{\partial W_{21}}{\partial x_{3}}
$$

then (42) can be rewritten as

$$
\frac{\partial \gamma_{2}}{\partial x_{1}}-\frac{\partial \gamma_{1}}{\partial x_{2}}=0 \text { in } \mathcal{D}^{\prime}(\Omega)
$$

By using the extension of Poincaré's Lemma provided in [23], we conclude that, for almost every $x_{3} \in(0, \ell)$, there exists $\varphi\left(\cdot, x_{3}\right) \in H^{1}(\omega)$ such that

$$
\frac{\partial \varphi}{\partial x_{1}}=\gamma_{1} \quad \text { and } \quad \frac{\partial \varphi}{\partial x_{2}}=\gamma_{2}
$$

whence (41).

\section{$3 \Gamma$-convergence and asymptotic behaviour of the scaled Korn's constant}

We find it convenient to reformulate the minization problem (21) in terms of the functional $R^{\varepsilon}: L^{2}\left(\Omega ; \mathbb{R}^{3}\right) \times L^{2}\left(\Omega ; \mathbb{R}^{3 \times 3}\right) \rightarrow \mathbb{R} \cup\{+\infty\}$ defined by:

$$
R^{\varepsilon}(\boldsymbol{v}, \boldsymbol{H}):= \begin{cases}\int_{\Omega}\left|\boldsymbol{E}^{\varepsilon}(\boldsymbol{v})\right|^{2} \mathrm{~d}^{3} \boldsymbol{x} & \text { if }(\boldsymbol{v}, \boldsymbol{H}) \in V \text { and } \int_{\Omega}|\boldsymbol{H}|^{2} \mathrm{~d}^{3} \boldsymbol{x}=1, \\ +\infty & \text { otherwise }\end{cases}
$$

where

$$
V:=\left\{(\boldsymbol{v}, \boldsymbol{H}) \in H_{\#}^{1}\left(\Omega ; \mathbb{R}^{3}\right) \times L^{2}\left(\Omega ; \mathbb{R}^{3 \times 3}\right): \boldsymbol{H}=\boldsymbol{H}^{\varepsilon}(\boldsymbol{v})\right\} .
$$

It is readily seen that

$$
\kappa^{\varepsilon}=\min R^{\varepsilon} .
$$

The next two propositions show that $R^{\varepsilon} \Gamma$-converges in the space $L^{2}\left(\Omega ; \mathbb{R}^{3}\right) \times L^{2}\left(\Omega ; \mathbb{R}^{3 \times 3}\right)$ to the functional

$$
R(\boldsymbol{v}, \boldsymbol{W}):= \begin{cases}\int_{\Omega}\left(\frac{\partial v_{3}}{\partial x_{3}}\right)^{2}+\frac{J_{t}}{2|\omega|}\left(\frac{\partial W_{21}}{\partial x_{3}}\right)^{2} \mathrm{~d}^{3} \boldsymbol{x} & \text { if }(\boldsymbol{v}, \boldsymbol{W}) \in V_{B N} \text { and } \int_{\Omega}|\boldsymbol{W}|^{2} \mathrm{~d}^{3} \boldsymbol{x}=1 \\ +\infty & \text { otherwise }\end{cases}
$$

where $J_{t}$ is the constant defined in (8) and

$$
V_{B N}:=\left\{(\boldsymbol{v}, \boldsymbol{W}) \in H_{\#}^{1}\left(\Omega ; \mathbb{R}^{3}\right) \times H_{\#}^{1}\left(\Omega ; \mathbb{R}_{\mathrm{skw}}^{3 \times 3}\right): \frac{\partial \boldsymbol{W}}{\partial x_{\alpha}}=0, E_{\alpha i}(\boldsymbol{v})=0, W_{\alpha 3}(\boldsymbol{v})=W_{\alpha 3}\right\} .
$$

In the last definition, the meaning of the subscript \# is the same as in (17). 
Proposition 3 (Lower bound). Let $\left\{\left(\boldsymbol{v}^{\varepsilon}, \boldsymbol{H}^{\varepsilon}\right)\right\} \subset L^{2}\left(\Omega ; \mathbb{R}^{3}\right) \times L^{2}\left(\Omega ; \mathbb{R}^{3 \times 3}\right)$ be a sequence such that $\left(\boldsymbol{v}^{\varepsilon}, \boldsymbol{H}^{\varepsilon}\right) \stackrel{L^{2}}{\rightarrow}(\boldsymbol{v}, \boldsymbol{W})$. Then

$$
R(\boldsymbol{v}, \boldsymbol{W}) \leq \liminf _{\varepsilon \rightarrow 0} R^{\varepsilon}\left(\boldsymbol{v}^{\varepsilon}, \boldsymbol{H}^{\varepsilon}\right) .
$$

Proof. We assume, without loss of generality, that $R^{\varepsilon}\left(\boldsymbol{v}^{\varepsilon}, \boldsymbol{H}^{\varepsilon}\right) \leq C$. Then, $\int_{\Omega}\left|\boldsymbol{H}^{\varepsilon}\right|^{2} \mathrm{~d}^{3} \boldsymbol{x}=1$, and hence, by passing to the limit,

$$
\int_{\Omega}|\boldsymbol{W}|^{2} \mathrm{~d}^{3} \boldsymbol{x}=1
$$

Futhermore, we have $\int_{\Omega}\left|\boldsymbol{E}^{\varepsilon}\left(\boldsymbol{v}^{\varepsilon}\right)\right|^{2} \mathrm{~d}^{3} \boldsymbol{x} \leq C$ and $\boldsymbol{H}^{\varepsilon}\left(\boldsymbol{v}^{\varepsilon}\right)=\boldsymbol{H}^{\varepsilon}$, and hence, by Propositions 1 and 2 ,

$$
\boldsymbol{E}^{\varepsilon}\left(\boldsymbol{v}^{\varepsilon}\right) \stackrel{L^{2}}{\rightarrow} \boldsymbol{E}, \quad E_{33}=\frac{\partial v_{3}}{\partial x_{3}}, \quad(\boldsymbol{v}, \boldsymbol{W}) \in V_{B N} .
$$

By (47) and (48) $)_{3}$, we have

$$
R(\boldsymbol{v}, \boldsymbol{W})=\int_{\Omega}\left(\frac{\partial v_{3}}{\partial x_{3}}\right)^{2}+\frac{J_{t}}{2|\omega|}\left(\frac{\partial W_{21}}{\partial x_{3}}\right)^{2} \mathrm{~d}^{3} \boldsymbol{x} .
$$

Moreover, by $(48)_{1}$,

$$
\begin{aligned}
\liminf _{\varepsilon \rightarrow 0} R\left(\boldsymbol{v}^{\varepsilon}, \boldsymbol{H}^{\varepsilon}\right) & =\liminf _{\varepsilon \rightarrow 0} \int_{\Omega}\left|\boldsymbol{E}^{\varepsilon}\left(\boldsymbol{v}^{\varepsilon}\right)\right|^{2} \mathrm{~d}^{3} \boldsymbol{x} \geq \int_{\Omega}|\boldsymbol{E}|^{2} \mathrm{~d}^{3} \boldsymbol{x} \\
& \geq \int_{\Omega} E_{33}^{2}+2\left(E_{13}^{2}+E_{23}^{2}\right) \mathrm{d}^{3} \boldsymbol{x} .
\end{aligned}
$$

By (41), (48) ${ }_{2}$ and (50), we have

$$
\liminf _{\varepsilon \rightarrow 0} R\left(\boldsymbol{v}^{\varepsilon}, \boldsymbol{H}^{\varepsilon}\right) \geq \int_{\Omega}\left(\frac{\partial v_{3}}{\partial x_{3}}\right)^{2}+\frac{1}{2}\left|x^{\perp} \frac{\partial W_{21}}{\partial x_{3}}+\nabla_{x} \varphi\right|^{2} \mathrm{~d}^{3} \boldsymbol{x} .
$$

Now, let $\psi_{\mathrm{m}}$ be the solution of (10). We observe that

$$
\int_{\omega}\left|x^{\perp} \frac{\partial W_{21}}{\partial x_{3}}+\nabla_{x} \varphi\right|^{2} \mathrm{~d}^{2} x \geq\left(\frac{\partial W_{21}}{\partial x_{3}}\right)^{2} \int_{\omega}\left|x^{\perp}+\nabla \psi_{\mathrm{m}}\right|^{2} \mathrm{~d}^{2} x=\left(\frac{\partial W_{21}}{\partial x_{3}}\right)^{2} J_{t}
$$

for a.e. $x_{3} \in(0, \ell)$. The above inequality is trivial if $\frac{\partial W_{21}}{\partial x_{3}}\left(x_{3}\right)=0$; if, instead, $\frac{\partial W_{21}}{\partial x_{3}}\left(x_{3}\right) \neq 0$, it is recovered by setting $\varphi=\frac{\partial W_{21}}{\partial x_{3}}\left(x_{3}\right) \psi$ and by minimizing with respect to $\psi$, bearing in mind (8) and (9). Integrating with respect to $x_{3}$, we obtain

$$
\frac{1}{2} \int_{\Omega}\left|x^{\perp} \frac{\partial W_{21}}{\partial x_{3}}+\nabla_{x} \varphi\right|^{2} \mathrm{~d}^{3} \boldsymbol{x} \geq \frac{J_{t}}{2|\omega|} \int_{\Omega}\left(\frac{\partial W_{21}}{\partial x_{3}}\right)^{2} \mathrm{~d}^{3} \boldsymbol{x} .
$$

By putting together (49), (51), and (52) we obtain the thesis. 
Proposition 4 (Upper bound). For every $(\boldsymbol{v}, \boldsymbol{W}) \in L^{2}\left(\Omega ; \mathbb{R}^{3}\right) \times L^{2}\left(\Omega ; \mathbb{R}^{3 \times 3}\right)$ there exists a sequence $\left\{\left(\boldsymbol{v}^{\varepsilon}, \boldsymbol{H}^{\varepsilon}\right)\right\} \subset L^{2}\left(\Omega ; \mathbb{R}^{3}\right) \times L^{2}\left(\Omega ; \mathbb{R}^{3 \times 3}\right)$ such that

$$
\left(\boldsymbol{v}^{\varepsilon}, \boldsymbol{H}^{\varepsilon}\right) \stackrel{L^{2}}{\rightarrow}(\boldsymbol{v}, \boldsymbol{W}) \text { and } \limsup _{\varepsilon \rightarrow 0} R^{\varepsilon}\left(\boldsymbol{v}^{\varepsilon}, \boldsymbol{H}^{\varepsilon}\right) \leq R(\boldsymbol{v}, \boldsymbol{W}) .
$$

Proof. We restrict our attention to the same case considered in the proof of Proposition 1, i.e., $\#=d d$, the other cases being similar. Let $(\boldsymbol{v}, \boldsymbol{W}) \in L^{2}\left(\Omega ; \mathbb{R}^{3}\right) \times L^{2}\left(\Omega ; \mathbb{R}^{3 \times 3}\right)$ be such that $R(\boldsymbol{v}, \boldsymbol{W})<+\infty$. Then $(\boldsymbol{v}, \boldsymbol{W}) \in V_{B N}$,

$$
\boldsymbol{W}=\left(\begin{array}{ccc}
0 & -\theta & W_{13}(\boldsymbol{v}) \\
\theta & 0 & W_{23}(\boldsymbol{v}) \\
W_{31}(\boldsymbol{v}) & W_{31}(\boldsymbol{v}) & 0
\end{array}\right)
$$

and

$$
R(\boldsymbol{v}, \boldsymbol{W})=\int_{\Omega}\left(\frac{\partial v_{3}}{\partial x_{3}}\right)^{2}+\frac{J_{t}}{2|\omega|}\left(\frac{\partial \theta}{\partial x_{3}}\right)^{2} \mathrm{~d}^{3} \boldsymbol{x} .
$$

Let $\left\{\theta^{\varepsilon}\right\} \subset C_{0}^{\infty}(0, \ell)$ be a sequence such that

$$
\left\|\theta^{\varepsilon}-\theta\right\|_{H^{1}(0, \ell)} \rightarrow 0 \quad \text { and } \quad \varepsilon\left\|\theta^{\varepsilon}\right\|_{H^{2}(0, \ell)} \rightarrow 0,
$$

and let $\psi_{\mathrm{m}} \in H^{1}(\omega)$ be the function defined in (10). Moreover, let

$$
\overline{\boldsymbol{v}}^{\varepsilon}:=\boldsymbol{v}+\varepsilon \theta^{\varepsilon}\left(-x_{2} \boldsymbol{e}_{1}+x_{1} \boldsymbol{e}_{2}\right)+\varepsilon \frac{\partial \theta^{\varepsilon}}{\partial x_{3}} \psi_{\mathrm{m}} \boldsymbol{e}_{3}, \quad \overline{\boldsymbol{H}}^{\varepsilon}:=\boldsymbol{H}^{\varepsilon}\left(\overline{\boldsymbol{v}}^{\varepsilon}\right) .
$$

Then,

$$
\boldsymbol{E}^{\varepsilon}\left(\overline{\boldsymbol{v}}^{\varepsilon}\right)=\left(\frac{\partial v_{3}}{\partial x_{3}}+\varepsilon \psi_{\mathrm{m}} \frac{\partial^{2} \theta^{\varepsilon}}{\partial x_{3}^{2}}\right) \boldsymbol{e}_{3} \otimes \boldsymbol{e}_{3}+\frac{\partial \theta^{\varepsilon}}{\partial x_{3}} \operatorname{sym} \boldsymbol{\Gamma}
$$

and

$$
\overline{\boldsymbol{H}}^{\varepsilon}=\boldsymbol{W}-2\left(\theta^{\varepsilon}-\theta\right) \operatorname{skw}\left(\boldsymbol{e}_{1} \otimes \boldsymbol{e}_{2}\right)+\varepsilon \frac{\partial \theta^{\varepsilon}}{\partial x_{3}} \boldsymbol{\Gamma}+\varepsilon\left(\frac{\partial v_{3}}{\partial x_{3}}+\varepsilon \frac{\partial^{2} \theta^{\varepsilon}}{\partial x_{3}^{2}} \psi_{\mathrm{m}}\right) \boldsymbol{e}_{3} \otimes \boldsymbol{e}_{3},
$$

where we have set $\boldsymbol{\Gamma}:=\left(\begin{array}{ccc}0 & 0 & -x_{2} \\ 0 & 0 & x_{1} \\ \frac{\partial \psi_{\mathrm{m}}}{\partial x_{1}} & \frac{\partial \psi_{\mathrm{m}}}{\partial x_{2}} & 0\end{array}\right)$. With these definitions it follows that $\left(\overline{\boldsymbol{v}}^{\varepsilon}, \overline{\boldsymbol{H}}^{\varepsilon}\right) \stackrel{L^{2}}{\rightarrow}(\boldsymbol{v}, \boldsymbol{W})$ in $L^{2}\left(\Omega ; \mathbb{R}^{3}\right) \times L^{2}\left(\Omega ; \mathbb{R}^{3 \times 3}\right)$, and recalling (9), we deduce

$$
\lim _{\varepsilon \rightarrow 0} \int_{\Omega}\left|\boldsymbol{E}^{\varepsilon}\left(\overline{\boldsymbol{v}}^{\varepsilon}\right)\right|^{2} \mathrm{~d}^{3} \boldsymbol{x}=\int_{\Omega}\left(\frac{\partial v_{3}}{\partial x_{3}}\right)^{2}+\left(\frac{\partial \theta}{\partial x_{3}}\right)^{2}|\operatorname{sym} \boldsymbol{\Gamma}|^{2} \mathrm{~d}^{3} \boldsymbol{x}=R(\boldsymbol{v}, \boldsymbol{W}) .
$$

We next define:

$$
\boldsymbol{v}^{\varepsilon}:=\frac{\overline{\boldsymbol{v}}^{\varepsilon}}{\left\|\overline{\boldsymbol{H}}^{\varepsilon}\right\|} \quad \text { and } \quad \boldsymbol{H}^{\varepsilon}:=\frac{\overline{\boldsymbol{H}}^{\varepsilon}}{\left\|\overline{\boldsymbol{H}}^{\varepsilon}\right\|_{L^{2}}} .
$$


Since $\overline{\boldsymbol{H}}^{\varepsilon} \stackrel{L^{2}}{\rightarrow} \boldsymbol{W}$ and $\|\boldsymbol{W}\|_{L^{2}}=1$, we have also that $\left\|\overline{\boldsymbol{H}}^{\varepsilon}\right\|_{L^{2}} \rightarrow 1$. Since $\int_{\Omega}\left|\boldsymbol{E}^{\varepsilon}\left(\boldsymbol{v}^{\varepsilon}\right)\right|^{2} \mathrm{~d}^{3} \boldsymbol{x}=$ $R^{\varepsilon}\left(\boldsymbol{v}^{\varepsilon}, \boldsymbol{H}^{\varepsilon}\right)$ from (54) we have

$$
\left(\boldsymbol{v}^{\varepsilon}, \boldsymbol{H}^{\varepsilon}\right) \stackrel{L^{2}}{\rightarrow}(\boldsymbol{v}, \boldsymbol{W}) \quad \text { and } \quad \lim _{\varepsilon \rightarrow 0} R^{\varepsilon}\left(\boldsymbol{v}^{\varepsilon}, \boldsymbol{H}^{\varepsilon}\right)=R(\boldsymbol{v}, \boldsymbol{W}),
$$

as required.

The next result follows from the thory of $\Gamma$-convergence, see [10]. For completeness we give a proof.

Theorem 1. Let $\left\{\left(\boldsymbol{v}^{\varepsilon}, \boldsymbol{H}^{\varepsilon}\right)\right\} \subset L^{2}\left(\Omega ; \mathbb{R}^{3}\right) \times L^{2}\left(\Omega ; \mathbb{R}^{3 \times 3}\right)$ be a sequence such that

$$
R^{\varepsilon}\left(\boldsymbol{v}^{\varepsilon}, \boldsymbol{H}^{\varepsilon}\right) \leq \kappa^{\varepsilon}+\zeta(\varepsilon)
$$

where $\zeta(\varepsilon) \rightarrow 0$ as $\varepsilon \rightarrow 0$. Then there exists a subsequence (not relabeled) such that

$$
\boldsymbol{v}^{\varepsilon} \stackrel{L^{2}}{\rightarrow} \boldsymbol{v}, \quad \boldsymbol{H}^{\varepsilon} \stackrel{L^{2}}{\rightarrow} \boldsymbol{W}
$$

and

$$
\kappa^{\varepsilon} \rightarrow \kappa=R(\boldsymbol{v}, \boldsymbol{W})=\min R .
$$

Proof. It is easily seen that $\sup _{\varepsilon} R^{\varepsilon}\left(\boldsymbol{v}^{\varepsilon}, \boldsymbol{H}^{\varepsilon}\right)<+\infty$, thus by Proposition 1 we have that $\boldsymbol{v}^{\varepsilon} \stackrel{L^{2}}{\rightarrow} \boldsymbol{v}$, and $\boldsymbol{H}^{\varepsilon} \stackrel{L^{2}}{\rightarrow} \boldsymbol{W}$. By Proposition 3 and (56) it follows that

$$
R(\boldsymbol{v}, \boldsymbol{W}) \leq \liminf _{\varepsilon \rightarrow 0} R^{\varepsilon}\left(\boldsymbol{v}^{\varepsilon}, \boldsymbol{H}^{\varepsilon}\right) \leq \liminf _{\varepsilon \rightarrow 0} \kappa^{\varepsilon} .
$$

Let $(\overline{\boldsymbol{v}}, \overline{\boldsymbol{W}}) \in L^{2}\left(\Omega ; \mathbb{R}^{3}\right) \times L^{2}\left(\Omega ; \mathbb{R}^{3 \times 3}\right)$. By Proposition 4 there exists a sequence $\left\{\left(\overline{\boldsymbol{v}}^{\varepsilon}, \overline{\boldsymbol{H}}^{\varepsilon}\right)\right\} \subset$ $L^{2}\left(\Omega ; \mathbb{R}^{3}\right) \times L^{2}\left(\Omega ; \mathbb{R}^{3 \times 3}\right)$ such that $\left(\overline{\boldsymbol{v}}^{\varepsilon}, \overline{\boldsymbol{H}}^{\varepsilon}\right) \stackrel{L^{2}}{\rightarrow}(\overline{\boldsymbol{v}}, \overline{\boldsymbol{W}})$ and

$$
\limsup _{\varepsilon \rightarrow 0} \kappa^{\varepsilon} \leq \limsup _{\varepsilon \rightarrow 0} R^{\varepsilon}\left(\overline{\boldsymbol{v}}^{\varepsilon}, \overline{\boldsymbol{H}}^{\varepsilon}\right) \leq R(\overline{\boldsymbol{v}}, \overline{\boldsymbol{W}}) .
$$

From (58) and (59) it immediately follows that

$$
R(\boldsymbol{v}, \boldsymbol{W}) \leq \liminf _{\varepsilon \rightarrow 0} \kappa^{\varepsilon} \leq \limsup _{\varepsilon \rightarrow 0} \kappa^{\varepsilon} \leq R(\overline{\boldsymbol{v}}, \overline{\boldsymbol{W}}),
$$

for every $(\overline{\boldsymbol{v}}, \overline{\boldsymbol{W}}) \in L^{2}\left(\Omega ; \mathbb{R}^{3}\right) \times L^{2}\left(\Omega ; \mathbb{R}^{3 \times 3}\right)$. Hence $R(\boldsymbol{v}, \boldsymbol{W})=\min R=\kappa$, and taking $(\overline{\boldsymbol{v}}, \overline{\boldsymbol{W}})=(\boldsymbol{v}, \boldsymbol{W})$ we deduce that $\lim _{\varepsilon \rightarrow 0} \kappa^{\varepsilon}=\kappa$.

\section{Characterization of minima and minimizers of the $\Gamma$-limit}

As shown in Theorem 1, see the previous section, the computation of the limit $\kappa$ boils down to the minimization of the $\Gamma$-limit defined in (44). In this section, we compute explicitly the minimum of the $\Gamma$-limit, and we show that:

$$
\kappa=\frac{1}{2|\omega|} \min \left\{\frac{J_{t}}{2} c_{\#}^{(0)}, J_{1} c_{\#}^{(1)}, J_{2} c_{\#}^{(1)}\right\}, \quad \#=d d, d n, n n,
$$


where,

$$
c_{\#}^{(0)}=\min _{\theta \in H_{\#}^{1}(0, \ell)} \frac{\int_{0}^{\ell}\left|\theta^{\prime}\right|^{2} \mathrm{~d} x_{3}}{\int_{0}^{\ell}|\theta|^{2} \mathrm{~d} x_{3}}, \quad c_{\#}^{(1)}=\min _{w \in H_{\#}^{2}(0, \ell)} \frac{\int_{0}^{\ell}\left|w^{\prime \prime}\right|^{2} \mathrm{~d} x_{3}}{\int_{0}^{\ell}\left|w^{\prime}\right|^{2} \mathrm{~d} x_{3}},
$$

and

$$
\begin{aligned}
& H_{d d}^{1}(0, \ell)=\left\{f \in H^{1}(0, \ell): f(0)=f(\ell)=0\right\}, \quad H_{d n}^{1}(0, \ell)=\left\{f \in H^{1}(0, \ell): f(0)=0\right\} \\
& H_{n n}^{1}(0, \ell)=\left\{f \in H^{1}(0, \ell): \int_{0}^{\ell} f \mathrm{~d} x_{3}=0\right\}, \\
& H_{d d}^{2}(0, \ell)=\left\{f \in H^{2}(0, \ell): f(0)=f(\ell)=f^{\prime}(0)=f^{\prime}(\ell)=0\right\}, \\
& H_{d n}^{2}(0, \ell)=\left\{f \in H^{2}(0, \ell): f(0)=f^{\prime}(0)=0\right\}, \\
& H_{n n}^{2}(0, \ell)=\left\{f \in H^{2}(0, \ell): \int_{0}^{\ell} f \mathrm{~d} x_{3}=\int_{0}^{\ell} f^{\prime} \mathrm{d} x_{3}=0\right\} .
\end{aligned}
$$

Poincaré's constants defined in (61) can be explicitly computed by solving the EulerLagrange equation corresponding to each variation problem, or by means of results from Fourier Analysis, adapting an argument from [11, p. 47]. One finds:

$$
\begin{aligned}
& c_{d d}^{(0)}=\left(\frac{\pi}{\ell}\right)^{2}, \quad c_{d d}^{(1)}=\left(\frac{2 \pi}{\ell}\right)^{2} \\
& c_{d n}^{(0)}=c_{d n}^{(1)}=\left(\frac{\pi}{2 \ell}\right)^{2}, \quad c_{n n}^{(0)}=c_{n n}^{(1)}=\left(\frac{\pi}{\ell}\right)^{2} .
\end{aligned}
$$

In order to establish (60), let us consider a pair $(\boldsymbol{v}, \boldsymbol{W}) \in L^{2}\left(\Omega ; \mathbb{R}^{3}\right) \times L^{2}\left(\Omega ; \mathbb{R}^{3 \times 3}\right)$ such that $R(\boldsymbol{v}, \boldsymbol{W})<+\infty$. Then, by $(44)$,

$$
R(\boldsymbol{v}, \boldsymbol{W})=\int_{\Omega}\left(\frac{\partial v_{3}}{\partial x_{3}}\right)^{2}+\frac{J_{t}}{2|\omega|}\left(\frac{\partial W_{21}}{\partial x_{3}}\right)^{2} \mathrm{~d}^{3} \boldsymbol{x},
$$

and $(\boldsymbol{v}, \boldsymbol{W}) \in V_{B N}$. Thus,

$$
E_{\alpha i}(\boldsymbol{v})=0, \quad W_{\alpha 3}(\boldsymbol{v})=W_{\alpha 3}, \quad \int_{\Omega}|\boldsymbol{W}|^{2} \mathrm{~d}^{3} \boldsymbol{x}=1,
$$

and there exists $\theta \in H_{\#}^{1}(0, \ell)$ such that

$$
W_{21}(\boldsymbol{x})=\theta\left(x_{3}\right)
$$

Therefore,

$$
R(\boldsymbol{v}, \boldsymbol{W})=\int_{\Omega}\left(\frac{\partial v_{3}}{\partial x_{3}}\right)^{2} \mathrm{~d}^{3} \boldsymbol{x}+\frac{J_{t}}{2} \int_{0}^{\ell}\left(\theta^{\prime}\right)^{2} \mathrm{~d} x_{3} .
$$

Next, we invoke the following result from [28, Section 4.1]: 
Proposition 5. Let $\boldsymbol{v} \in H_{\#}^{1}\left(\Omega ; \mathbb{R}^{3}\right)$ satisfy $E_{\alpha i}(\boldsymbol{v})=0$. Then there exist $w_{\alpha} \in H_{\#}^{2}(0, \ell)$ and $w_{3} \in H_{\#}^{1}(0, \ell)$ such that

$$
\boldsymbol{v}(\boldsymbol{x})=\boldsymbol{w}\left(x_{3}\right)-x_{\alpha} w_{\alpha}^{\prime}\left(x_{3}\right) \boldsymbol{e}_{3}
$$

for all $\boldsymbol{x} \in \Omega$.

By $(64)_{2,3},(65)$, and (67), we have

$$
1=\int_{\Omega}|\boldsymbol{W}|^{2} \mathrm{~d}^{3} \boldsymbol{x}=2|\omega| \int_{0}^{\ell} w_{1}^{\prime 2}+w_{2}^{\prime 2}+\theta^{2} \mathrm{~d} x_{3},
$$

and, on recalling (7),

$$
\int_{\Omega}\left(\frac{\partial v_{3}}{\partial x_{3}}\right)^{2} \mathrm{~d}^{3} \boldsymbol{x}=\int_{0}^{\ell} J_{2} w_{1}^{\prime \prime 2}+J_{1} w_{2}^{\prime \prime 2}+|\omega| w_{3}^{\prime 2} \mathrm{~d} x_{3}
$$

(here we use the choice of $x_{1}$ and $x_{2}$ as the principal centroidal axes of the cross section, see (16)).

Using (66), (69), and (61), we have

$$
\begin{aligned}
R(\boldsymbol{v}, \boldsymbol{W}) & =\int_{0}^{\ell} J_{2} w_{1}^{\prime \prime 2}+J_{1} w_{2}^{\prime \prime 2}+|\omega| w_{3}^{\prime 2}+\frac{J_{t}}{2}\left(\theta^{\prime}\right)^{2} \mathrm{~d} x_{3} \\
& \geq J_{2} \int_{0}^{\ell}\left(w_{1}^{\prime \prime}\right)^{2} \mathrm{~d} x_{3}+J_{1} \int_{0}^{\ell}\left(w_{2}^{\prime \prime}\right)^{2} \mathrm{~d} x_{3}+\frac{J_{t}}{2} \int_{0}^{\ell}\left(\theta^{\prime}\right)^{2} \mathrm{~d} x_{3} \\
& \geq J_{2} c_{\#}^{(1)} \int_{0}^{\ell}\left(w_{1}^{\prime}\right)^{2} \mathrm{~d} x_{3}+J_{1} c_{\#}^{(1)} \int_{0}^{\ell}\left(w_{2}^{\prime}\right)^{2} \mathrm{~d} x_{3}+\frac{J_{t}}{2} c_{\#}^{(0)} \int_{0}^{\ell} \theta^{2} \mathrm{~d} x_{3} \\
& \geq \min \left\{\frac{J_{t}}{2} c_{\#}^{(0)}, J_{1} c_{\#}^{(1)}, J_{2} c_{\#}^{(1)}\right\} \int_{0}^{\ell} \theta^{2}+\left(w_{1}^{\prime}\right)^{2}+\left(w_{2}^{\prime}\right)^{2} \mathrm{~d} x_{3} .
\end{aligned}
$$

We note on passing that (13) follows from the first line of the above chain. Taking into account (68), we arrive at

$$
R(\boldsymbol{v}, \boldsymbol{W}) \geq \frac{1}{2|\omega|} \min \left\{\frac{J_{t}}{2} c_{\#}^{(0)}, J_{1} c_{\#}^{(1)}, J_{2} c_{\#}^{(1)}\right\} .
$$

We next show that the lower bound (74) is attained for some $(\overline{\boldsymbol{v}}, \overline{\boldsymbol{W}})$. To this aim, we pick two functions $g \in H_{\#}^{1}(0, \ell)$ and $h \in H_{\#}^{2}(0, \ell)$ such that

$$
\begin{array}{ll}
\int_{0}^{\ell}\left|g^{\prime}\right|^{2} \mathrm{~d} x_{3}=c_{\#}^{(0)}, & \int_{0}^{\ell}|g|^{2} \mathrm{~d} x_{3}=1, \\
\int_{0}^{\ell}\left|h^{\prime \prime}\right|^{2} \mathrm{~d} x_{3}=c_{\#}^{(1)}, & \int_{0}^{\ell}\left|h^{\prime}\right|^{2} \mathrm{~d} x_{3}=1 .
\end{array}
$$

Then, for

$$
\bar{\theta}=A g, \quad \bar{w}_{1}=B h, \quad \bar{w}_{2}=C h, \quad \bar{w}_{3}=0,
$$


we define

$$
\overline{\boldsymbol{v}}(\boldsymbol{x}):=\overline{\boldsymbol{w}}\left(x_{3}\right)-x_{\alpha} \bar{w}_{\alpha}^{\prime}\left(x_{3}\right) \boldsymbol{e}_{3}, \quad \overline{\boldsymbol{W}}:=\left(\begin{array}{ccc}
0 & -\bar{\theta} & \bar{w}_{1}^{\prime} \\
\bar{\theta} & 0 & \bar{w}_{2}^{\prime} \\
-\bar{w}_{1}^{\prime} & -\bar{w}_{2}^{\prime} & 0
\end{array}\right)
$$

(cf. (67), (64) $)_{2}$, and (65)). We then have $(\overline{\boldsymbol{v}}, \overline{\boldsymbol{W}}) \in V_{B N}$. Moreover, we have $\int_{\Omega}|\overline{\boldsymbol{W}}|^{2} \mathrm{~d}^{3} \boldsymbol{x}=$ $2|\omega|\left(A^{2}+B^{2}+C^{2}\right)$. Thus, provided that

$$
A^{2}+B^{2}+C^{2}=\frac{1}{2|\omega|}
$$

we have

$$
R(\overline{\boldsymbol{v}}, \overline{\boldsymbol{W}})=c_{\#}^{(0)} \frac{J_{t}}{2} A^{2}+c_{\#}^{(1)} J_{2} B^{2}+c_{\#}^{(1)} J_{1} C^{2} .
$$

By performing a minimization of the right-hand side of (77) with the constraint (76), we find the optimal choice for $A, B, C$ :

$$
\begin{cases}A^{2}=\frac{1}{2|\omega|}, B=C=0 & \text { if } \frac{J_{t}}{2} c_{\#}^{(0)}<\min \left\{J_{1} c_{\#}^{(1)}, J_{2} c_{\#}^{(1)}\right\} \\ B^{2}=\frac{1}{2|\omega|}, A=C=0 & \text { if } J_{1} c_{\#}^{(1)}<\min \left\{\frac{J_{t}}{2} c_{\#}^{(0)}, J_{2} c_{\#}^{(1)}\right\} \\ C^{2}=\frac{1}{2|\omega|}, B=C=0 & \text { otherwise. }\end{cases}
$$

With this choice,

$$
R(\overline{\boldsymbol{v}}, \overline{\boldsymbol{W}})=\frac{1}{2|\omega|} \min \left\{\frac{J_{t}}{2} c_{\#}^{(0)}, J_{1} c_{\#}^{(1)}, J_{2} c_{\#}^{(1)}\right\},
$$

so that the lower bound in (74) is attained, and (60) is established.

We now write (60) explicitly. We first discuss case $(d d)$. To this aim, it is useful to note that $J_{t} \leq \int_{\omega}\left|x^{\perp}+\nabla_{x} \psi\right|^{2} \mathrm{~d}^{2} x$, for every $\psi \in H^{1}(\omega)$. In particular, by taking $\psi=x_{1} x_{2}$ and $\psi=-x_{1} x_{2}$, we have

$$
\frac{J_{t}}{2} \leq 2 \min \left\{J_{1}, J_{2}\right\}
$$

Because of (62) and (79), we see that

$$
\frac{J_{t}}{2} c_{d d}^{(0)}=\frac{J_{t}}{2}\left(\frac{\pi}{\ell}\right)^{2} \leq 2\left(\frac{\pi}{\ell}\right)^{2} \min \left\{J_{1}, J_{2}\right\}<\left(\frac{2 \pi}{\ell}\right)^{2} \min \left\{J_{1}, J_{2}\right\}=\min \left\{J_{1} c_{d d}^{(1)}, J_{2} c_{d d}^{(1)}\right\} .
$$

Thus, by (60)

$$
\kappa=\frac{1}{2|\omega|} \frac{J_{t}}{2} c_{d d}^{(0)}=\frac{1}{2|\omega|}\left(\frac{\pi}{\ell}\right)^{2} \frac{J_{t}}{2} \quad \text { for case }(d d) .
$$

For case $(d n)$, we have by (60) and (62)

$$
\kappa=\frac{1}{2|\omega|} \min \left\{\frac{J_{t}}{2} c_{d n}^{(0)}, J_{1} c_{d n}^{(1)}, J_{2} c_{d n}^{(1)}\right\}=\frac{1}{2|\omega|}\left(\frac{\pi}{2 \ell}\right)^{2} \min \left\{\frac{J_{t}}{2}, J_{1}, J_{2}\right\} .
$$


For case $(n n)$, we have, instead,

$$
\kappa=\frac{1}{2|\omega|} \min \left\{\frac{J_{t}}{2} c_{n n}^{(0)}, J_{1} c_{n n}^{(1)}, J_{2} c_{n n}^{(1)}\right\}=\frac{1}{2|\omega|}\left(\frac{\pi}{\ell}\right)^{2} \min \left\{\frac{J_{t}}{2}, J_{1}, J_{2}\right\} .
$$

We have thus proven (11a)-(11b).

Remarkably, the limit $\kappa$ is always ruled by $J_{t}$ in the case $(d d)$, that is, the critical mode is torsional, see the discussion after Remark 2 below, leading to (81). The next couple of examples show that in the cases $(d n)$ and $(n n)$ the minimum in (11a) may be determined either by $J_{t} / 2$ or by $J_{1}$ and $J_{2}$.

Remark 1. For an ellipsoidal cross-section with semi-axes $a$ and $b$ we have

$$
J_{1}=\frac{\pi a b^{3}}{4}, \quad J_{2}=\frac{\pi a^{3} b}{4}, \quad J_{t}=\frac{\pi a^{3} b^{3}}{a^{2}+b^{2}},
$$

see [51] (p. 121-122, where $J_{t}$ is denoted by $D / \mu$ ). Let $\lambda:=b / a$ be the ratio between the semi-axes. Then, for $\lambda \geq 1$ we have that $J_{2} \leq J_{1}$ and

$$
\frac{J_{t}}{J_{2}}=\frac{4}{1+1 / \lambda^{2}} \text {. }
$$

We first note that as $\lambda \rightarrow+\infty$ we have that $J_{t} / J_{2} \rightarrow 4$ and hence inequality (79) is sharp. Also, for $\lambda>1$ we deduce, from (80), that $J_{t} / J_{2}>2$, thus

$$
\kappa=\frac{1}{2|\omega|}\left(\frac{\pi}{2 \ell}\right)^{2} J_{2}=\frac{\pi^{2}}{32}\left(\frac{a}{\ell}\right)^{2},
$$

for case $(d n)$, while

$$
\kappa=\frac{1}{2|\omega|}\left(\frac{\pi}{\ell}\right)^{2} J_{2}=\frac{\pi^{2}}{8}\left(\frac{a}{\ell}\right)^{2}
$$

for case $(n n)$.

Remark 2. For a square cross-section of side $a$ we have

$$
J_{1}=J_{2}=\frac{a^{4}}{12}, \quad J_{t} \approx 1.68 \frac{a^{4}}{12},
$$

see [51] (p. 132). Thus $J_{t} / 2<J_{1}=J_{2}$, and hence

$$
\kappa=\frac{1}{2|\omega|}\left(\frac{\pi}{2 \ell}\right)^{2} \frac{J_{t}}{2} \approx \frac{\pi^{2}}{57.14}\left(\frac{a}{\ell}\right)^{2},
$$

for case $(d n)$, while

$$
\kappa=\frac{1}{2|\omega|}\left(\frac{\pi}{\ell}\right)^{2} \frac{J_{t}}{2} \approx \frac{\pi^{2}}{14.28}\left(\frac{a}{\ell}\right)^{2}
$$

for case $(n n)$. 
The fact that the critical mode in the case $(d d)$ is always torsional may appear counterintuitive to a reader conversant with elastic stability. For this reason, a brief sketch of the connection of our results with that theory is worthwhile. Let us think of $\Omega^{\varepsilon}$ as a homogeneous rod made of a isotropic material and subject to a uniform stress field, see [30, 52, 53], of the form:

$$
\stackrel{\circ}{\boldsymbol{T}}=-\Sigma \boldsymbol{e}_{3} \otimes \boldsymbol{e}_{3}, \quad \Sigma \geq 0 .
$$

The rod is infinitesimally stable if

$$
\int_{\Omega^{\varepsilon}} \mathbb{L}[\boldsymbol{E}(\boldsymbol{u})] \cdot \boldsymbol{E}(\boldsymbol{u})+\stackrel{\circ}{\boldsymbol{T}} \cdot \nabla \boldsymbol{u}^{T} \nabla \boldsymbol{u} \mathrm{d}^{3} \boldsymbol{x} \geq 0
$$

for every admissible displacement field $\mathbf{u}$, where $\mathbb{L}$ is the incremental elasticity tensor (see for instance $[30,46,47])$. Loss of stability occurs as soon as $\Sigma$ attains the value

$$
\Sigma^{\varepsilon}=\inf \frac{\int_{\Omega^{\varepsilon}} \mathbb{L}[\boldsymbol{E}(\boldsymbol{u})] \cdot \boldsymbol{E}(\boldsymbol{u}) \mathrm{d}^{3} x}{\int_{\Omega^{\varepsilon}} \boldsymbol{e}_{3} \otimes \boldsymbol{e}_{3} \cdot \nabla \boldsymbol{u}^{T} \nabla \boldsymbol{u} \mathrm{d}^{3} x} .
$$

By using the approach of the present paper, one could prove that $\Sigma^{\varepsilon}$ scales as $\varepsilon^{2}$, and that, as $\varepsilon$ tends to zero, the scaled multipler $\sigma^{\varepsilon}=\Sigma^{\varepsilon} / \varepsilon^{2}$ converges to a limit $\sigma$. In particular, if $\mathbb{L}$ is an isotropic tensor, the limit $\sigma$ is the minimum of

$$
\mathcal{S}(\boldsymbol{w}, \theta)=\frac{\int_{0}^{\ell}|\omega| E w_{3}^{\prime 2}+E J_{2} w_{1}^{\prime \prime 2}+E J_{1} w_{2}^{\prime \prime 2}+G J_{t} \theta^{\prime 2} \mathrm{~d} x_{3}}{|\omega| \int_{0}^{\ell} w_{1}^{\prime 2}+w_{2}^{\prime 2} \mathrm{~d} x_{3}},
$$

where $E$ and $G$ are the Young modulus and the shear modulus. At variance with $\mathcal{R}$, the denominator of $\mathcal{S}$ does not contain $\theta$. In this case, minimizers satisfy $\theta=0$ and hence torsional modes are ruled out.

\section{Acknowledgements.}

The authors thank Patrizio Neff for valuable comments on the first draft of this manuscript. RP acknowledges the support given by Regione Autonoma della Sardegna, Legge 7/2007, Bando 2010, under the project "Modellazione Matematica nella Meccanica dei Materiali (M4)".

\section{References}

[1] Anzellotti, G., Baldo, S. and Percivale, D. Dimension reduction in variational problems, asymptotic development in $\Gamma$-convergence and thin structures in elasticity. Asymptotic Anal., 9, 61-100 (1994). 
[2] Bernstein, B. and Toupin, R. Korn inequalities for the sphere and circle. Arch. Rational Mech. Anal., 6, 51-64 (1960).

[3] Blanchard, D., Gaudiello, A. and Griso, G. Junction of a periodic family of elastic rods with a thin plate. Part I. J. Math. Pures Appl., 88, 1-33 (2007).

[4] Blanchard, D., Gaudiello, A. and Griso, G. Junction of a periodic family of elastic rods with a thin plate. Part II. J. Math. Pures Appl., 88, 149-190 (2007).

[5] Brenner, S. C. and Scott, L. R. The mathematical theory of finite element methods, 3rd edition, Springer, New York, 2008.

[6] Ciarlet, P. G. Plates and junctions in elastic multi-structures, Masson, Paris, 1990.

[7] Ciarlet, P. G. Mathematical elasticity. Vol. II. North-Holland, Amsterdam, 1997.

[8] Cioranescu, D., Oleinik, O. A. and Tronel, G. Korn's inequalities for frame type structures and junctions with sharp estimates for the constants. Asymptotic Anal., 8, 1-14 (1994).

[9] Dafermos, C. M. Some remarks on Korn's inequality. Z. Angew. Math. Phys., 19, 913-920 (1968).

[10] Dal Maso, G. An introduction to Г-convergence. Birkhäuser, Boston, 1993.

[11] Dym, H. and McKean, H. P. Fourier's series and integrals Academic Press, N.Y., 1972.

[12] Duvaut, G. and Lions, J.-L. Inequalities in mechanics and physics. Springer-Verlag, Berlin, 1976.

[13] Freddi, L., Mora, M.G. and Paroni, R. Nonlinear thin-walled beams with rectangular cross-section - Part I. Math. Models Methods Appl. Sci. 22, 1150016 (34 pp) (2012).

[14] Freddi, L., Morassi, A. and Paroni, R. Thin-walled beams: the case of the rectangular cross-section. J. Elasticity 76, 45-66 (2005).

[15] Freddi, L., Morassi, A. and Paroni R. Thin-walled beams: a derivation of Vlassov theory via $\Gamma$-convergence, J. Elasticity, 86, 263-296 (2007).

[16] Friedrichs, K. On the boundary-value problems of the theory of elasticity and Korn's inequality. Ann. of Math., 48, 441-471 (1947).

[17] Friesecke, G., James, R. D. and Müller, S. A hierarchy of plate models derived from nonlinear elasticity by $\Gamma$-convergence. Arch. Rational Mech. and Anal., 180, 183-236 (2006).

[18] Gaudiello, A., Monneau, R., Mossino, J., Murat F. and Sili, A. On the junction of elastic plates and beams. C. R. Math. Acad. Sci. Paris, 335, 717-722 (2002). 
[19] Geymonat, G. and Gilardi, G. Contre-exemples à l'inégalité de Korn et au lemme de Lions dans des domaines irréguliers. In Équations aux dérivées partielles et applications, pages 541-548. Gauthier-Villars, Éd. Sci. Méd. Elsevier, Paris, 1998.

[20] Grabovsky, Y. and Truskinovsky, L. The flip side of buckling. Cont. Mech. Thermodyn., 19, 211-243 (2007).

[21] Holden, J. T. Estimation of critical loads in elastic stability theory. Arch. Rational Mech. Anal., 17, 171-183 (1964).

[22] Horgan, C. O. Korn's inequalities and their applications in continuum mechanics. SIAM Rev., 37, 491-511 (1995).

[23] Kesavan, S. On Poincaré's and J.-L. Lions' lemmas. C. R. Math. Acad. Sci. Paris, 340, 27-30 (2005).

[24] Kohn R. V. and Vogelius, M. A new model for thin plates with rapidly varying thickness: II. A convergence proof. Quart. Appl. Math., 43, 1-22 (1985).

[25] V. A. Kondrat'ev and O. A. Oleinik. Boundary value problems for a system in elasticity theory in unbounded domains. Korn inequalities. Russ. Math. Surv., 43, 65-119 (1988).

[26] Kondrat'ev V. A. and Oleinik O. A. On the dependence of the constant in Korn's inequality on parameters characterizing the geometry of the region. Russ. Math. Surv., 44, 187-195 (1989).

[27] Korn, A. Die Eigenschwingungen eines elastischen Körpers mit ruhender Oberfläche. Akad. der Wissenschaften München, Math.-Phys. Klasse, Berichte 36, 351-401 (1906).

[28] Le Dret, H. Problèmes Variationnels dans les Multi-domaines: modélisation des Jonctions et Applications. Masson, Paris, 1991.

[29] Lewicka, M. and Müller, S. The uniform Korn-Poincaré inequality in thin domains. Ann. Inst. H. Poincaré Anal. Non Linéaire, 28, 443-469 (2011).

[30] Man, C. S. and Carlson, D. E. On the traction problem of dead loading in linear elasticity with residual stress. Arch. Rational Mech. Anal., 128, 223-247 (1994).

[31] Mosolov, P. P. and Mjasnikov, V. P. A proof of Korn's inequality. Dokl. Akad. Nauk SSSR, 201, 36-39 (1971).

[32] Nazarov, S. A. Korn inequalities that are asymptotically exact for thin domains. Vestnik S.-Peterburg. Univ. Mat., 2, 18-22 (1992).

[33] Nazarov, S. A. Korn's inequalities for junctions of spatial bodies and thin rods. Math. Methods Appl. Sci., 20, 219-243 (1997). 
[34] Neff, P. On Korn's first inequality with non-constant coefficients. Proc. Roy. Soc. Edinburgh: A, 132, 221-243 (2002).

[35] Neff, P. and Chełmiński, K. A geometrically exact Cosserat shell-model for defective elastic crystals. Justification via $\Gamma$-convergence. Interfaces and Free Boundaries, 9, 455-492 (2007).

[36] Neff, P., Hong, K.-I. and Jeong J. The Reissner-Mindlin plate is the $\Gamma$-limit of Cosserat elasticity. Math. Models Methods Appl. Sci., 20, 1553-1590 (2010).

[37] Neff, P., Pauly, D. and Witsch, K.-J. A canonical extension of Korn's first inequality to $\mathrm{H}(\mathrm{Curl})$ motivated by gradient plasticity with plastic spin. C. R. Math. Acad. Sci. Paris, 349, 1251-1254 (2011).

[38] Neff, P., Pauly, D. and Witsch K.J., Maxwell meets Korn: A new coercive inequality for tensor fields in $\mathbb{R}^{N \times N}$ with square integrable exterior derivative. Math. Meth. Appl. Sci., 35, 65-71 (2012).

[39] Nitsche, J. A. On Korn's second inequality. RAIRO Anal. Numér. , 15, 237-248 (1981).

[40] Oleinik, O. A., Shamaev, A. S. and Yosifian, G. A. Mathematical problems in elasticity and homogenization. North-Holland, 1992.

[41] Paroni, R. The equations of motion of a plate with residual stress. Meccanica, 41, 1-21, 2006.

[42] Paroni, R. Theory of linearly elastic residually stressed plates. Math. Mech. Solids, 11, 137-159, 2006.

[43] Paroni, R., Podio-Guidugli, P., and Tomassetti, G. The Reissner-Mindlin plate theory via $\Gamma$-convergence. C. R. Math. Acad. Sci. Paris, 343, 437-440, 2006.

[44] Paroni, R., Podio-Guidugli, P., and Tomassetti, G. A justification of the ReissnerMindlin plate theory through variational convergence. Anal. Appl., 5, 165-182, 2007.

[45] Paroni, R. and Tomassetti, G. Asymptotically exact Korn's constant for thin cylindrical domains. C. R. Math. Acad. Sci. Paris, accepted (2012).

[46] Paroni, R. and Tomassetti, G. A variational justification of linear elasticity with residual stress. J. Elasticity, 97, 189-206 (2009).

[47] Paroni, R. and Tomassetti, G. From non-linear elasticity to linear elasticity with initial stress via $\Gamma$-convergence. Contin. Mech. Thermodyn., 23, 347-361 (2011).

[48] Payne, L. E. and Weinberger, H. F. On Korn's inequality. Arch. Rational Mech. Anal., 8, 89-98 (1961). 
[49] Riey, G. and Tomassetti, G. A variational model for linearly elastic micropolar plate-like bodies. J. Convex Analysis, 15, 677-691 (2008).

[50] Riey, G. and Tomassetti, G. Micropolar linearly elastic rods. Commun. Appl. Anal., 13, 647-657 (2009).

[51] Sokolnikoff, I. S. Mathematical theory of elasticity, 2nd ed. McGraw-Hill, New York, 1956.

[52] Steigmann, D. J. Linear theory for the bending and extension of a thin, residually stressed, fiber-reinforced lamina. Int. J. Eng. Sci., 47, 1367-1378 (2009).

[53] Steigmann, D. J. Elastic waves interacting with a thin, prestressed, fiber-reinforced surface film. Int. J. Eng. Sci., 48, 1604-1609 (2010).

[54] Tiero, A. On Korn's inequality in the second case. J. Elasticity, 54, 187-191 (1999).

[55] Villaggio, P. Qualitative methods in elasticity. Noordhoff, Leiden, 1977.

[56] Weck, N. Local compactness for linear elasticity in irregular domains. Math. Meth. Appl. Sci., 17, 107-113 (1994). 